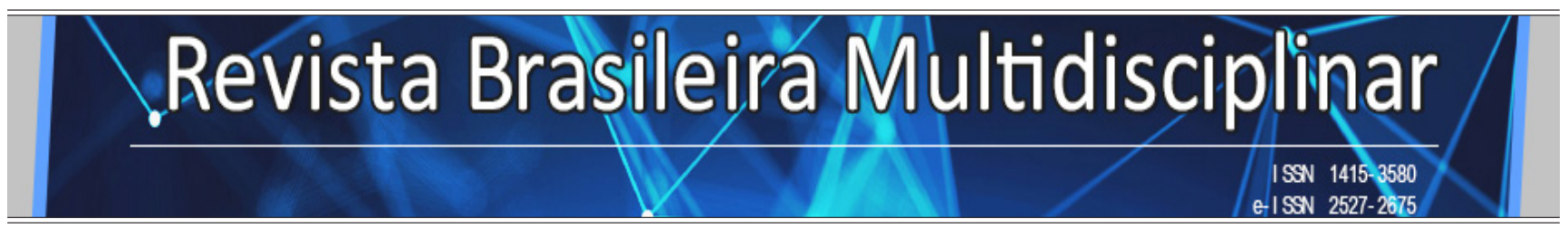

http://revistarebram.com/index.php/revistauniara

\title{
Recessão Gengival: Uma Revisão Narrativa
}

\section{Glayson Pereira Vitor}

*Professor adjunto do curso de Odontologia da Faculdade de Estudos Administrativos de Minas Gerais - FEAD. BH/MG. Mestre em Odontologia com ênfase em Periodontia (UFMG).

${ }^{*}$ Autor para correspondência e-mail: glayson.vitor@fead.br

\section{Palavras-chave}

Epidemiologia

Etiologia

Recessão Gengival

\section{KEYWORDS}

Epidemiology

Etiology

Gingival recession

\begin{abstract}
RESUMO
Recessão Gengival (RG) é definida como o deslocamento apical da margem gengival em relação à junção cemento-esmalte. Objetivo: verificar os possíveis fatores etiológicos e epidemiológicos que permeiam RG através de uma revisão narrativa. Metodologia: foram selecionados estudos transversais, longitudinais e revisões sistemáticas, indexados nas bases de dados do portal de periódico CAPES, MEDLINE e PubMed usando os descritores epidemiologia, etiologia e recessão gengival. Discussão: Levantamentos epidemiológicos e estudos longitudinais reportam que RG é altamente prevalente na população adulta, $88 \%$ das pessoas com idade $\geq 65$ anos e $50 \%$ entre 18 e 64 anos têm pelo menos um local com RG. Sua etiologia é multifatorial e com diferentes fatores relacionados que podem agir em associação, tais como: biofilme bacteriano, escovação traumática, fatores locais de retenção de placa, tabagismo e movimentação ortodôntica. Conclusão: O mecanismo na qual ocorre a RG ainda não é bem entendido sendo uma particularidade relatada na maioria das populações, tanto em países desenvolvidos quanto em subdesenvolvidos, constitui um evento comum na clínica odontológica, frequentemente percebida pelos indivíduos e levando a busca por orientações profissionais e tratamento.
\end{abstract}

\section{Abstract \\ GINGIVAL RECESSION: A NARRATIVE REVIEW}

Gingival recession (GR) is defined as the apical displacement of the gingival margin relative to the cement-enamel junction. Objective: The objective was to verify the possible etiological and epidemiological factors that permeate GR through a narrative review. Methods: Cross-sectional, longitudinal and systematic reviews indexed in CAPES, MEDLINE and PubMed databases were selected by using the descriptors epidemiology, etiology and gingival recession. Discussion: epidemiological surveillance and longitudinal studies report highly GR prevalence in the adult population; $88 \%$ of individuals aged $\geq 65$ years and $50 \%$ between 18 and 64 years old have GR in at least one spot. This manifestation's etiology is multifactorial and different related factors can act in association with it such as: bacterial biofilm, traumatic brushing, local plaque retention factors, smoking and orthodontic movement. Conclusion: The mechanism in which GR occurs is still not well understood and it is a condition present in most populations in developed and developing countries. GR is a common case in dental practices, often noted by the patients who seek for professional orientation and treatment. 


\section{INTRODUÇÃO}

Recessão gengival (RG) é o termo usado para definir a manifestação clínica da migração apical da gengiva marginal em relação à junção cemento-esmalte (KASSAB; COHEN, 2003; RIOS et al., 2014) deixando a superfície radicular exposta ao meio bucal. Corresponde, portanto, a uma perda de inserção com uma posição mais apical da gengiva marginal. Este processo de movimentação pode ocorrer em qualquer área exposta da superfície da raiz e se manifestar em ambas as arcadas, em qualquer face e em qualquer dente (MARINI et al., 2004; YARED; ZENOBIO; PACHECO, 2006).

O mecanismo na qual ocorre a RG ainda não é bem entendido (CORTELLINI; BISSADA, 2018), mas parece ser um processo de natureza inflamatória (SUSIN, 2004). Sua etiologia é multifatorial e com diferentes fatores relacionados que podem agir em associação. Dentre os fatores que podem desencadear o processo de recessão temos a escovação traumática (McCRACKEN, 2009), anatomia óssea (MAROSO, 2015), inserção do freio labial, posicionamento dentário, doença periodontal, fatores locais de retenção de placa, fumo e movimentação ortodôntica (ALBANDAR; KINGMAN, 1999; SUSIN, 2004).

No Brasil a prevalência de pelo menos um local com RG $\geq 1 \mathrm{~mm}$ é 29,5\% em jovens de 14 a 19 anos e 99\% em adultos mais de 40 anos (SUSIN et al., 2004). Diante disto e dos problemas clínicos associados com a RG, o objetivo deste estudo foi realizar uma revisão dos possíveis fatores etiológicos e epidemiológicos que permeiam RG, uma vez que o conhecimento dos resultados da literatura sobre fatores relacionados a RG contribui com a discussão acadêmica/científica e fornece aos cirurgiões-dentistas, clínicos ou especialistas, referências para o correto diagnóstico e tratamento destas alterações.

\section{Metodologia}

O estudo caracterizou-se como revisional, a partir de busca na literatura selecionando estudos transversais, longitudinais e revisões sistemáticas, em português e inglês, indexados nas bases de dados do portal de periódico CAPES, MEDLINE e PubMed. Utilizou os Descritores em Ciências da Saúde (DECS): "epidemiologia" e "etiologia" associados ao termo "recessão gengival" e/ou somente "recessão gengival”, os termos equivalentes em inglês também foram utilizados. Caso clínico, relato de caso e artigos de opinião foram excluídos. Primeiro foi realizado uma triagem pelo título seguida de leitura dos resumos, após constatar relação com o tema e objetivo da revisão, os artigos foram vistos na íntegra por um único pesquisador.

\section{Discussão}

RG é uma particularidade relatada na maioria das populações, tanto em países desenvolvidos quanto em subdesenvolvidos, ocorrendo em indivíduos com boa ou má higienização bucal (LÖE; ANERUD; BOYSEN, 1992; CHAMBRONE; TATAKIS, 2016). Estima-se que mais da metade da população dos Estados Unidos apresentam RG (ALBANDAR, 2002) e levantamentos epidemiológicos e estudos longitudinais reportam que RG é altamente prevalente na população adulta (SARFATI et al., 2010), sendo que, aproximadamente $88 \%$ das pessoas com idade $\geq 65$ anos e $50 \%$ entre 18 e 64 anos têm pelo menos um sítio com RG. (CORTELLINI; BISSADA, 2018).

No Brasil, um alto índice de RG também tem sido reportado, entretanto com taxas de prevalência e gravidade variadas. Foi demonstrado que aproximadamente $99 \%$ dos adultos com idade acima de 40 anos apresentam RG, e em torno de 50\% desses indivíduos esta RG é $\geq 3 \mathrm{~mm}$ (SUSIN et al., 2004). Outro relato demonstrou que aproximadamente $75 \%$ dos indivíduos com idade superior a 35 anos apresentavam pelo menos um dente com RG em torno de $3 \mathrm{~mm}$ e cerca de $40 \%$ apresentavam RG de aproximadamente 5 $\mathrm{mm}$ (RIOS et al., 2014). 
VITOR

Observa-se que sua ocorrência tem uma alta variação nos estudos, chegando a flutuar entre 3 a $100 \%$. Os resultados dependem da população estudada, do critério de diagnóstico utilizado e do método de análise dos dados (LITONJUA et al., 2003; MAROSO et al., 2015). Entretanto, estima-se que 60\% da população humana apresentem RG (DOMINIAK; GEDRANGE, 2014). Sendo assim, a RG é um evento comum na clínica odontológica, frequentemente percebida pelos indivíduos e levando a busca por orientações profissionais e tratamento (TOKER; OZDEMIR, 2009; NIERI et al., 2013; MYTHRI et al., 2015), especialmente quando afeta os dentes anteriores (CHRYSANTHAKOPOULOS, 2013). Além de levar a um comprometimento estético, a RG pode propiciar abrasões, lesões de cárie, hipersensibilidade, retenção de placa e ainda influenciar negativamente a qualidade de vida do indivíduo ( SARFATI et al., 2010; RIOS et al., 2014; GOH; CORBET; LEUNG, 2016; WAGNER et al., 2016).

Não há um consenso na literatura com relação aos dentes que são mais afetados pela RG (MARINI et al., 2004). Entretanto, alguns relatos têm apontado uma maior ocorrência de RG em incisivos inferiores e primeiros molares seguidos dos caninos e pré molares superiores (RAJAPAKSE et al., 2007; MCCRACKEN et al., 2009).

A RG parece ser um processo de natureza inflamatória (SUSIN et al., 2004; CHRYSANTHAKOPOULOS, 2013). A partir de uma inflamação localizada, o epitélio do sulco pode proliferar em direção vestibular, invadir o cório gengival e anastomosar-se com o epitélio oral. A invasão epitelial resulta em uma diminuição da área ocupada pelo tecido conjuntivo, fazendo com que a porção marginal do epitélio sofra necrose por falta de nutrição. A descamação sem substituição por novas células pode causar a diminuição da superfície epitelial, produzindo clinicamente a RG (TUGNAIT; CLEREHUGH, 2001; CHRYSANTHAKOPOULOS, 2013). Deste modo, sugere-se que a RG é influenciada pelo fenótipo periodontal, resultante da combinação de fenótipo gengival com a espessura do osso alveolar vestibular (CATON, 2018).

Entretanto, sua etiologia vem sendo demonstrada por diversos estudos como multifatorial, envolvendo diferentes variáveis relacionadas a processos anatômicos, patológicos e fisiológicos (MARINI et al., 2004; MCCRACKEN et al., 2009; CHRYSANTHAKOPOULOS, 2013; DÖRFER; STAEHLE; WOLFF, 2016) e que podem agir em associação com variáveis sociodemográficas e hábitos comportamentais (RIOS et al., 2014). Contudo, tem sido relatadas dificuldades em se identificar quais variáveis são mais importantes na sua causalidade (KASSAB; BADAWI; DENTINO, 2010; MAROSO et al., 2015).

Alguns autores dividem estas variáveis em dois grupos, definidos como predisponentes e precipitantes (MARINI et al., 2004; JATI; FURQUIM; CONSOLARO, 2016). As predisponentes são tidas como aquelas de características anatômicas locais que favorecem a ocorrência da alteração gengival, como anatomia óssea, deiscência óssea e quantidade de gengiva inserida (MAROSO et al., 2015; ZUCHELLI; MOUNSSIF, 2015), erupção e posicionamento dentário, inserção de freio e oclusão traumática (MARINI et al., 2004; KRISHNA PRASAD; SRIDHAR SHETTY; SOLOMON, 2013). Já as variáveis precipitantes são aquelas que levam ao aparecimento da RG, ou seja, responsáveis pela indução ou ativação da RG. As mais mencionadas na literatura referem-se ao biofilme bacteriano, escovação traumática (MCCRACKEN et al., 2009; MYTHRI et al., 2015; DÖRFER; STAEHLE; WOLFF, 2016), fatores locais de retenção de placa, tabagismo, movimentação ortodôntica (ALBANDAR; KINGMAN, 1999; SUSIN et al., 2004; PRASHANT et al., 2014;) e periodontite ( LÖE; ANERUD; BOYSEN, 1992; CHRYSANTHAKOPOULOS, 2013).

Estudos têm demonstrado que a prevalência, extensão e gravidade da RG tendem a aumentar gradativamente com a idade (KASSAB; COHEN, 2003; SUSIN et al., 2004; RIOS et al., 2014), sugerindose o efeito acumulativo, de um longo período de exposição do complexo mucogengival aos possíveis agentes etiológicos da RG, associados às mudanças fisiológicas locais e sistêmicas com o passar dos anos (MYTHRI et al., 2015). A idade pode aumentar a probabilidade de ocorrência da RG, mas isto não significa que seja uma característica advinda do envelhecimento (JATI; FURQUIM; CONSOLARO, 2016). 
A ocorrência da RG em indivíduos jovens é frequentemente relatada como mais localizada e associada a fatores etiológicos isolados, como por exemplo, escovação de forma incorreta e com excesso de força. Em indivíduos mais velhos aparece com um padrão mais generalizado, envolvendo com maior frequência áreas interproximais (MARINI et al., 2004; MYTHRI et al., 2015).

Estudos relatam que homens apresentam maiores níveis de RG em relação às mulheres (SERINO et al., 1994; MARINI et al., 2004; SARFATI et al., 2010; CHANG, 2012; DOMINIAK; GEDRANGE, 2014; RIOS et al., 2014; WAGNER et al., 2016). No Brasil, foi reportado que aproximadamente $85 \%$ dos homens apresentavam RG de $\geq 3 \mathrm{~mm}$ em pelo menos um dente, comparados a 75\% das mulheres (RIOS et al., 2014). Entretanto, outros estudos não encontraram associação significativa entre sexo e RG (SUSIN et al., 2004; CHRYSANTHAKOPOULOS, 2013).

Alguns estudos reportam que a RG pode estar associada ao acúmulo localizado do biofilme bacteriano (SERINO et al., 1994; VAN PALENSTEIN HELDERMAN et al., 1998; SUSIN et al., 2004; ZUCHELLI; MOUNSSIF, 2015) e cálculo dental (ALBANDAR; KINGMAN, 1999; SARFATI et al., 2010). Fatores locais retentores de placa também podem estar associados a RG, como restaurações mal adaptadas, que podem não somente resultar em trauma direto nos tecidos periodontais, mas ao mesmo tempo facilitar o acúmulo de biofilme (ZUCHELLI; MOUNSSIF, 2015). É interessante observar que alguns autores apontam que o acúmulo de biofilme tende a ser uma consequência da RG (JOSHIPURA; KENT; DEPAOLA, 1994; MARINI et al., 2004; YARED; ZENOBIO; PACHECO, 2006).

O controle mecânico do biofilme microbiano através da escovação, de forma rotineira, é de suma importância para a saúde periodontal (LÖE; ANERUD; BOYSEN, 1992; MYTHRI et al., 2015). Entretanto, a escovação traumática é usualmente associada a RG e explica parcialmente a correlação entre os baixos níveis de biofilme bacteriano encontrados em sítios de RG (RAJAPAKSE et al., 2007). Estudos relatam que dados para apoiar ou refutar a associação entre escovação dentária e recessão gengival são inconclusivos (HEASMAN et al, 2017; CORTELLINI, BISSADA, 2018). O trauma é geralmente causado por uma escovação inadequada, principalmente com utilização das cerdas na horizontal, excesso de força, tempo excessivo de escovação inadequada, cerdas da escova muito rígidas e creme dental abrasivo (RAJAPAKSE et al., 2007; ZUCHELLI; MOUNSSIF, 2015).

O uso inadequado do fio dental também pode contribuir para a abrasão e lesões nos tecidos gengivais. Estas lesões ocorrem frequentemente em pacientes altamente motivados que não foram devidamente instruídos na técnica de uso do fio dental (WALTERS; CHANG, 2003; ZUCHELLI; MOUNSSIF, 2015). A RG associada ao uso do fio dental pode ser mais prevalente em regiões interproximais, enquanto a RG associada a hábitos deletérios de escovação, em áreas vestibulares ( LÖE; ANERUD; BOYSEN, 1992; ALBANDAR; KINGMAN, 1999; LITONJUA et al., 2003; MARINI et al., 2004).

Não há evidências de que o tratamento ortodôntico possa ser um fator primário as recessões gengivais, apesar de levar os dentes envolvidos a situações que ajam como fatores predisponentes para que as causas diretas possam atuar e produzir recessões, principalmente quando o tratamento deixa-se uma tábua óssea vestibular muito fina ou, até, com deiscência (JATI, FURQUIM, CONSOLARO 2016; MORRIS et al, 2017;). Entretanto, existe a possibilidade de início da recessão gengival ou progressão da recessão durante ou após o tratamento ortodôntico, a prevalência relatada é de $5 \%$ a $12 \%$ no final do tratamento (CORTELLINI, BISSADA, 2018).

O tabagismo é considerado uma importante variável associada a RG ( SUSIN et al., 2004; SARFATI et al., 2010; CHRYSANTHAKOPOULOS, 2013; RIOS et al., 2014) . Foi reportado que o uso do cigarro está fortemente associado com a ocorrência de RG localizada e generalizada em indivíduos jovens e em indivíduos acima de 30 anos foi associado com a RG generalizada (SUSIN et al., 2004). O tabagismo pode 
VITOR

contribuir para um quadro menos favorável no processo de cicatrização periodontal, vasoconstrição, e diminuição de mecanismos de defesa contra microorganismos (ZUCHELLI; MOUNSSIF, 2015).

Evidências da alta prevalência de periodontite na América Latina, sugerem que a RG também seja prevalente nesta população (SUSIN et al., 2004), uma vez que a periodontite tem sido a variável mais importante associada a RG (CHRYSANTHAKOPOULOS, 2013). Neste sentido, alguns autores relatam uma associação direta entre gravidade da periodontite e a gravidade da RG (VAN DER VELDEN et al., 2006; SARFATI et al., 2010). A reação inflamatória em consequência do biofilme dental é uma característica biológica predominante e compartilhada pela RG e pela periodontite (SARFATI et al., 2010), levando à migração apical do epitélio e à destruição do ligamento periodontal juntamente com a reabsorção óssea (DENTINO et al., 2013). A RG, como consequência do processo de doença periodontal, pode ocorrer em diferentes cenários (TUGNAIT; CLEREHUGH, 2001; KASSAB; COHEN, 2003) . Tratamentos periodontais também podem estar associados a RG (TUGNAIT; CLEREHUGH, 2001).

\section{CoNCLUSÃo}

RG está associada à perda de inserção e à exposição da superfície radicular ao meio bucal, ocorre com frequência em adultos, tem tendência a aumentar com a idade e é diagnosticada tanto em indivíduos com bom ou mal índice de higiene bucal. Embora a etiologia da RG permaneça incerta, o que sugere a necessidade contínua de estudos, vários fatores predisponentes têm sido sugeridos, os mais mencionados na literatura referem-se ao biofilme bacteriano, escovação traumática, fatores locais de retenção de placa, tabagismo e movimentação ortodôntica.

\section{REFERÊNCIAS}

ALBANDAR, JM. Global risk factors and risk indicators for periodontal diseases. Periodontology 2000, v. 29, n. 1, p. 177-206, 2002.

ALBANDAR, JM; KINGMAN, A. Gingival Recession, Gingival Bleeding, and Dental Calculus in adults 30 years of Age and Older in the United States , 1988-1994. J Periodontol, v. 70, n. 1, p. 30-43, 1999.

CATON GJ, ARMITAGE G, BERGLUNDH T, CHAPPLE ILC, JEPSEN S, KORNMAN KS, et al. A new classification scheme for periodontal and peri-implant diseases and conditions - Introduction and key changes from the 1999 classification. J Clin Periodontol. V.45(Suppl 20), p.1-8, 2018.

CHAMBRONE, L; TATAKIS, DN. Long-term Outcomes of Untreated Buccal Gingival Recessions: A Systematic Review and Meta-Analysis. J Periodontol, v. 87, n. 7, p. 1-17, 2016.

CHANG, LC. Comparison of Age and Sex Regarding Gingival and Papillary Recession. The International Journal of Periodontics \& Restorative Dentistry, v. 32, n. 5, p. 555-561, 2012.

CHRYSANTHAKOPOULOS, NA. Prevalence and associated factors of gingival recession in Greek adults. Journal of Investigative and Clinical Dentistry, v. 4, n. 3, p. 178-185, 2013.

CORTELLINI P, BISSADA NF. Mucogingival conditions in the natural dentition: Narrative review, case definitions, and diagnostic considerations. J Clin Periodontol. v. 45(Suppl 20), p.190-8, 2018. 
Recessão Gengival: Uma Revisão Narrativa...

DENTINO, A et al. Principles of periodontology. Periodontology 2000, v. 61, n. 1, p. 16-53, 2013.

DÖRFER, CE; STAEHLE, HJ; WOLFF, D. Three-year randomized study of manual and power toothbrush effects on pre-existing gingival recession. J Clin Periodontol, v. 43, n. 6, p. 512-519, 2016.

DOMINIAK, M; GEDRANGE, T. New Perspectives in the Diagnostic of Gingival Recession. Advances in Clinical and Experimental Medicine, v. 23, n. 6, p. 857-863, 2014.

GOH, V; CORBET, EF; LEUNG, WK. Impact of dentine hypersensitivity on oral health-related quality of life in individuals receiving supportive periodontal care. J Clin Periodontol, v. 43, n. 7, p. 595-602, 2016.

HEASMAN PA, RITCHIE M, ASUNI A, GAVILLET E, SIMONSEN JL, NYVAD B. GINGIVAL recession and root caries in the ageing population: a critical evaluation of treatments. J Clin Periodontol. v.44(Suppl 18), p.178-193, 2017.

JATI AS, FURQUIM LZ, CONSOLARO A. Gingival recession: its causes and types, and the importance of orthodontic treatment. Dental Press J Orthod. v 21, n 3, p. 18-29, 2017.

JOSHIPURA, KJ; KENT, RL; DEPAOLA, PF. Gingival recession: intra-oral distribution and associated factors. J Periodontol, v. 65, n. 9, p. 864-871, 1994.

KASSAB, MM; BADAWI, H; DENTINO, AR. Treatment of Gingival Recession. Dental Clinics of North America, v. 54, n. 1, p. 129-140, 2010.

KASSAB, MM; COHEN, RE. The etiology and prevalence of gingival recession. J Am Dent Assoc, v. 134, n. 2, p. 220-225, 2003.

KRISHNA PRASAD, D; SRIDHAR SHETTY, N; SOLOMON, EGR. The influence of occlusal trauma on gingival recession and gingival clefts. Journal of Indian Prosthodontist Society, v. 13, n. 1, p. 7-12, 2013.

LITONJUA, LA et al. Toothbrushing and gingival recession. International Dental Journal, v. 53, n. 2, p. $67-72,2003$.

LÖE, H; ANERUD, A; BOYSEN, H. The natural history of periodontal disease in man: prevalence, severity, and extent of gingival recession. J Periodontol, v. 63, n. 6, p. 489-495, 1992.

MARINI, MG et al. Gingival recession: prevalence, extension and severity adults. J Appl Oral Sci, v. 12, n. 3, p. 250-255, 2004.

MAROSO, FB et al. Correlation between gingival thickness and gingival recession in humans. Acta Odontol Latinoam., v. 28, n. 2, p. 162-166, 2015.

MCCRACKEN, GI et al. The impact of powered and manual toothbrushing on incipient gingival recession. J Clin Periodontol, v. 36, n. 11, p. 950-957, 2009.

MORRIS JW, CAMPBELL PM, TADLOCK LP, BOLEY J, BUSCHANG PH. Prevalence of gingival reces- 
VITOR

sion after orthodontic tooth movements Am J Orthod Dentofacial Orthop. V.151, n.5, p. 851-859, 2017

MYTHRI, S et al. Etiology and occurrence of gingival recession - An epidemiological study. Journal of Indian Society of Periodontology, v. 19, n. 6, p. 671-675, 2015.

NIERI, $M$ et al. Patient perceptions of buccal gingival recessions and requests for treatment. J Clin Periodontol, v. 40, n. 7, p. 707-712, 2013.

PRASHANT, B et al. Classification \& prevalence of dental surface defects in areas of gingival recession - A clinical study. Journal of Clinical and Diagnostic Research, v. 8, n. 7, p. 1-4, 2014.

RAJAPAKSE, PS et al. Does tooth brushing influence the development and progression of non-inflammatory gingival recession? A systematic review: Review Article. J Clin Periodontol, v. 34, n. 12, p. 1046-1061, 2007.

RIOS, FS et al. Estimates and multivariable risk assessment of gingival recession in the population of adults from Porto Alegre, Brazil. J Clin Periodontol, v. 41, n. 11, p. 1098-1107, 2014.

SARFATI, A et al. Risk assessment for buccal gingival recession defects in an adult population. J Periodontol, v. 81, n. 10, p. 1419-1425, 2010.

SERINO, G et al. The prevalence and distribution of gingival recession in subjects with a high standard of oral hygiene. J Clin Periodontol, v. 21, n. 1, p. 57-63, 1994.

SUSIN, C et al. Gingival recession: epidemiology and risk indicators in a representative urban Brazilian population. J Periodontol, v. 75, n. 10, p. 1377-1386, 2004.

TOKER, H; OZDEMIR, H. Gingival recession: epidemiology and risk indicators in a university dental hospital in Turkey. International Journal of Dental Hygiene, v. 7, n. 2, p. 115-120, 2009.

TUGNAIT, A; CLEREHUGH, V. Gingival recession - its significance and management. Journal of Dentistry, v. 29, n. 6, p. 381-394, 2001.

VAN DER VELDEN, U. et al. Java project on periodontal diseases. The natural development of periodontitis: Risk factors, risk predictors and risk determinants. J Clin Periodontol, v. 33, n. 8, p. 540-548, 2006.

VAN PALENSTEIN HELDERMAN, W $\mathrm{H}$ et al. Gingival recession and its association with calculus in subjects deprived of prophylactic dental care. J Clin Periodontol, v. 25, n. 2, p. 106-111, 1998.

WAGNER, TP et al. Gingival recession and oral health-related quality of life: a population-based crosssectional study in Brazil. Community Dentistry and Oral Epidemiology, v. 44, n. 4, p. 390-399, 2016.

WALTERS, J D; CHANG, E I. Periodontal bone loss associated with an improper Flossing technique: case report. International Journal of Dental Hygiene, v. 1, n. 3, p. 115-119, 2003.

YARED, KFG; ZENOBIO, EG; PACHECO, W. A etiologia multifatorial da recessão periodontal. R Dental 
Recessão Gengival: Uma Revisão Narrativa...

Press Ortodon Ortop Facial, v. 11, n. 6, p. 45-51, 2006.

ZUCHELLI, G; MOUNSSIF, I. Periodontal plastic surgery. Periodontology 2000, v. 68, n. 1, p. 333-368, 2015. 